\title{
ASCA OBSERVATIONS OF LUMINOUS INFRARED GALAXIES
}

Evolution from Starburst to AGN?

\author{
TAKAO NAKAGAWA, TSUNEO KII, RYUICH FUJIMOTO, \\ TOSHIYUKI MIYAZAKI AND HAJIME INOUE \\ Institute of Space $\&$ Astronautical Science \\ 3-1-1 Yoshinodai, Sagamihara, Kanagawa 229, Japan \\ YASUSHI OGASAKA AND KEITH ARNAUD \\ NASA/GSFC, Laboratory for High Energy Astrophysics \\ Greenbelt, MD 20771, USA \\ AND \\ RYOHEI KAWABE \\ Nobeyama Radio Observatory \\ Nobeyama, Minamimaki, Minamisaku, Nagano 384-13, Japan
}

\section{Introduction}

\subsection{ORIGINS OF LUMINOSITY}

One of the most important results of the IRAS survey is the discovery of a class of "Luminous Infrared Galaxies" (LIGs), which emit most of the energy in the infrared and are the dominant population in the local universe at luminosities above $10^{11} L_{\odot}$ (e.g., Sanders \& Mirabel 1996). All LIGs appear to be extremely rich in molecular gas, and many of them show evidence of recent interacting/merging activities. Hence it is now accepted that strong interactions of gas-rich galaxies triggers large central concentration of molecular gas, and makes optimal conditions for both enormous nuclear starbursts and building and/or fueling AGN. Actually, various observations show evidence of starburst activity as well as that of AGN in many LIGs (Sanders \& Mirabel 1996).

Some of the "warm" (large $f_{\nu}(25 \mu m) / f_{\nu}(60 \mu m)$ ratio) LIGs (e.g., Mrk 231) show the closest appearance of classical quasars, such as broad emission lines and dominant optical nucleus. On the basis of these results, Sanders et al. (1988) proposed an evolutional connection between Ultra Luminous Infrared Galaxies (ULIGs, $L>10^{12} L_{\odot}$ ) and optical quasars. They 
identified ULIGs with cold infrared colors $\left(f_{\nu}(25 \mu m) / f_{\nu}(60 \mu m)<0.2\right)$ as the initial, dust-enshrouded stage, and "warm" $\left(f_{\nu}(25 \mu m) / f_{\nu}(60 \mu m)>\right.$ $0.2)$ ULIGs as a transition stage to optical quasars.

\subsection{WHY HARD X-RAY?}

It has been controversial what is the dominant source of luminosity of LIGs. However, heavy dust extinction in LIGs makes it very difficult to determine how much of the total luminosity is due to starbursts and how much can be attributed to AGN.

Here we present a preliminary result of our on-going program to make hard X-ray observations of LIGs. The hard X-ray observations have the following merits.

1. We can observe heavily obscured objects with $N_{\mathrm{H}}$ as large as $10^{24} \mathrm{~cm}^{-2}$

2. Hard X-ray observations are especially sensitive to AGN activities. The $L_{\mathrm{X}} / L_{\text {bol }}$ ratio is $\sim 0.1$ for Seyfert 1 type objects but is $10^{-4}$ for starburst galaxies (Kii et al. 1997).

3. Seyfert 1 type objects have a power-law spectrum with a photon index $\sim 1.7$, and starburst galaxies show a soft spectrum characteristic of hot thin plasm. The difference of the spectrum enables us to make quantitative estimates of contributions of AGN and starburst activities.

Hence the hard X-ray observations are indispensable to reveal the origins of the luminosities in LIGs.

\section{Results}

We observed 10 LIGs (Table 1) by the Japanese X-ray satellite $A S C A$. The Hubble constant $H_{0}$ is assumed to be $75 \mathrm{~km} \mathrm{~s}^{-1} \mathrm{Mpc}^{-1}$ to derive the luminosities. We saw clear evidence of AGN activities in 5 of the observed galaxies.

The most clear example is NGC 6240 . Its soft (below $2 \mathrm{keV}$ ) X-ray spectrum is well represented by optically thin hot plasma emission, which is probably due to starburst activity. On the other hand, the spectrum above $3 \mathrm{keV}$ is represented by a combination of flat power-law continuum and a complex Fe K line feature with very large equivalent width (total $\sim 2 \mathrm{keV}$ ). This spectrum is very similar to that of the archetypical Seyfert 2 galaxy NGC 1068 (Ueno et al. 1994). Hence we conclude that, in NGC 6240, the direct emission from the AGN is completely blocked by a Compton thick $\left(N_{\mathrm{H}}>10^{24} \mathrm{~cm}^{-2}\right)$ molecular torus and we see only a reflected component. The observed luminosity of the power-law component is $5 \times 10^{8} L_{\odot}$. Assuming that $\Omega / 4 \pi \sim 0.25$ ( $\Omega$ is the unobscured solid angle), we estimate that the intrinsic luminosity of the AGN component in NGC 6240 is $\sim 10^{11} L_{\odot}$, 
TABLE 1. Observed Luminous Infrared Galaxies (LIGs)

\begin{tabular}{lccrrr}
\hline Name & $z$ & $\log \left(\frac{L_{\mathrm{FIR}}}{L_{\odot}}\right)$ & $\frac{f_{\nu}(25 \mu \mathrm{m})}{f_{\nu}(60 \mu \mathrm{m})}$ & $\log \left(\frac{L_{2-10 \mathrm{keV}}}{L_{\odot}}\right)^{(1)}$ & $\frac{N_{\mathrm{H}}}{10^{22} \mathrm{~cm}^{-2}}$ \\
\hline Arp 220 & 0.018 & 11.9 & 0.08 & $<8.02^{(2)}$ & $\ldots$ \\
NGC 6240 & 0.024 & 11.5 & 0.15 & $10.89^{(3)}$ & $>100$ \\
Mrk 273 & 0.037 & 11.8 & 0.11 & $9.14^{(4)}$ & 20 \\
UGC 05101 & 0.039 & 12.0 & 0.09 & $<8.32^{(5)}$ & $\ldots$ \\
Mrk 231 & 0.042 & 12.1 & 0.27 & $8.89^{(4)}$ & 6 \\
IRAS 05189-2524 & 0.042 & 11.7 & 0.25 & $9.88^{(4)}$ & 5 \\
IRAS 08572+3915 & 0.058 & 12.1 & 0.23 & $8.26^{(4)}$ & $\ldots$ \\
IRAS 07598+6508 & 0.149 & 12.4 & 0.32 & $<9.02^{(5)}$ & $\ldots$ \\
IRAS 20460+1925 & 0.181 & 11.8 & 0.52 & $10.48^{(6)}$ & 3 \\
IRAS 15307+3252 & 0.926 & 12.8 & 0.55 & $<10.61^{(6)}$ & $\ldots$ \\
\hline
\end{tabular}

(1) Power law component with photon index of around 1.7

(2) Corrected for extinction with $N_{\mathrm{H}}=10^{23} \mathrm{~cm}^{-2}$. (3) Estimated intrinsic luminosity under the assumption that only the reflected component is observed.

(4) Corrected for extinction.

(5) Observed upper limit. Not corrected for extinction.

(6) Ogasaka et al. (1997)

which is luminous enough to power the whole far-infrared luminosity of NGC 6240 . There is large uncertainty of this estimate of the intrinsic luminosity of NGC 6240 , but we can conclude that a significant fraction of the total luminosity of NGC 6240 is due to the AGN activity.

We have detected clear sign of AGN activity in four more galaxies: IRAS 20460+1925 (Ogasaka et al. 1997), Mrk 273, IRAS 05189-2524, and Mrk 231. All of them show large extinction of $N_{\mathrm{H}}=3-20 \times 10^{22} \mathrm{~cm}^{-2}$. Even Mrk 231, which has been regarded as a Seyfert 1 galaxy, have large extinction which corresponds to $A_{\mathrm{v}} \sim 30 \mathrm{mag}$. The AGN contribution to the total luminosity is significant in IRAS $20460+1925$ and IRAS 05289-2524, but is small $(<10 \%)$ in Mrk 273 and Mrk 231.

In the most archetypical LIG Arp 220, we detected no hard X-ray emission but only weak soft X-ray emission. Even if we assume that the AGN is heavily obscured by $N_{\mathrm{H}}=10^{23} \mathrm{~cm}^{-2}$, the upper limit for the power-law component is $L_{2-10 \mathrm{keV}}<1 \times 10^{8} L_{\odot}$ or $L_{2-10 \mathrm{keV}} / L_{\mathrm{FIR}}<10^{-4}$. Hence we conclude that the contribution of AGN activity to the total luminosity in Arp 220 is negligible $(<1 \%)$ (if any).

\section{Discussion}

We plot the relative AGN contribution $\left(L_{2-10 \mathrm{keV}} / L_{\mathrm{FIR}}\right)$ versus the infrared color $\left(f_{\nu}(25 \mu \mathrm{m}) / f_{\nu}(60 \mu \mathrm{m})\right)$ in Figure 1. 


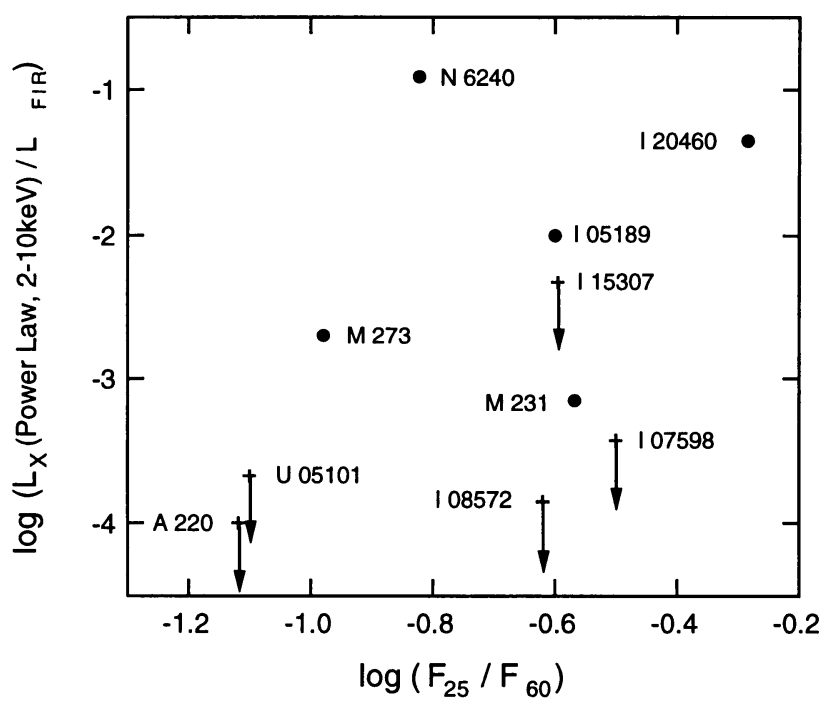

Figure 1. Infrared color $\left(f_{\nu}(25 \mu m) / f_{\nu}(60 \mu m)\right)$ versus relative AGN activity $\left(L_{2-10 \mathrm{keV}} / L_{\mathrm{FIR}}\right)$

Following the evolutionary scenario by Sanders et al (1988), we can expect a positive correlation between the two parameters. However, Figure 1 shows no obvious correlation between the two parameters. Moreover, we see no obvious trend for the AGN contribution to increase in the more advanced mergers (such as Mrk 231). We hence conclude that the proposed evolutionary scenario cannot be applied to all of the LIGs, and it is not the evolutional stage as mergers but the local accretion rate onto the central engine which determines the AGN activity.

\section{References}

Kii, T., Nakagawa, T., Fujimoto, R., Ogasaka, Y, Miyazaki, T., Kawabe, R. \& Terashima, Y. 1997, in X-Ray Imaging and Spectroscopy of Cosmic Hot Plasmas, ed. F. Makino \& K. Mitsuda (Tokyo: Universal Academy Press), 161

Ogasaka, Y., Inoue, H., Brandt, W., Fabian, A., Kii, T., Nakagawa, T., Fujimoto, R., \& Otani, C. 1997, PASJ, 49, 1790

Sanders, D. B., Soifer, B. T., Elias, J. H., Madore, B. F., Matthews, K., Neugebauer, G., \& Scoville, N. Z. 1988, ApJ, 325, 74

Sanders, D. B. \& Mirabel, I. F. 1996, ARAA, 34, 749

Ueno, S., Mushotzky, R. F., Koyama, K., Iwasawa, K., Awaki, H., \& Hayashi, I. 1994, PASJ, 46, L71 